\title{
COMUNICAÇÃO
}

\section{DESENVOLVIMENTO DE MUDAS DE AROEIRA (Schinus terebinthifolius) E SOMBREIRO (Clitoria fairchildiana) SOB CONDIÇÕES DE SOMBREAMENTO}

\author{
Development of Schinus terebinthifolius and Clitoria fairchildiana seedlings under shading
}

\author{
Silvana de Paula Quintão Scalon ${ }^{1}$, Rosilda Mara Mussury ${ }^{2}$, Homero Scalon Filho ${ }^{3}$, \\ Carla Silvana Fabro Francelino ${ }^{2}$
}

\begin{abstract}
RESUMO
Objetivou-se com este trabalho avaliar o desenvolvimento das mudas de Schinus terebinthifolius e Clitoria fairchildiana sob condições de sombreamento e pleno sol. As mudas foram cultivadas sob sombrites de $70 \%$ e $50 \%$ de luz e sob luz plena. O experimento foi conduzido em DBC com 4 repetições de 20 mudas. Foram avaliados a altura do caule e o diâmetro do colo a cada vinte e um dias. Ao final do experimento foram realizadas as avaliações de peso seco total (PST-g), área foliar (AF-dm²), razão de peso foliar (RPF -g/g); e peso específico de folha $\left(\mathrm{PEF}-\mathrm{g} / \mathrm{dm}^{2}\right)$. As sementes de aroeira do campo e do sombreiro apresentaram $70 \%$ e $85 \%$ de germinação, respectivamente. S.terebinthifolius tolera bem um sombreamento moderado (70\% de luz), sendo seu cultivo a pleno sol o mais indicado, pois é quando as mudas atingem menor altura $(32,93 \mathrm{~cm})$, diâmetro médio de 7,15 mm, maior PST (74,66 g); menor PEF (0,025); as mudas de $C$.fairchildiana sob condições de pleno sol não se desenvolveram satisfatoriamente, embora a altura não tenha variado entre os níveis de luz $(16,39 \mathrm{~cm})$, apresentaram menor diâmetro $(5,32 \mathrm{~mm})$, menor PST $(26,22 \mathrm{~g})$, menor AF $(32,98$ $\left.\mathrm{dm}^{2}\right)$ e maior PEF $(0,4)$.
\end{abstract}

Termos para indexação: Germinação, sombreamento, Fabaceae, Anacardiaceae.

\begin{abstract}
The objective of this work was to evaluate the development of $S$. terebinthifolius and $C$. fairchildiana seedlings under shade and full sunlight conditions. The seedlings were cultivated under $70 \%$ and $50 \%$ sunlight and full sunlight. The experiment was carried out in a randomized block design with four replications of twenty seedlings. Stem height and collar diameter were evaluated every 21 days. At the end of the experiment total dried weight (TDW-g), leaf area (LA-dm²), leaf weight rate LWR-g/g) and leaf specific weight (LSW-g/dm ${ }^{2}$ ) were also evaluated. The seeds of S.terebinthifolius and C. fairchildiana showed a germination of $70 \%$ and $85 \%$ respectively. $S$. terebinthifolius tolerated moderate shade (70\% sunligth) however, full sunlight seemed to be most appropriate. $C$. fairchildiana seedlings did not develop in a satisfactory manner under full sunlight. Although the stem height did not vary among light levels $(16.39 \mathrm{~cm})$, the seedlings showed lower diameter $(5.32 \mathrm{~mm})$, lower total dried weight $(26.22)$, lower leaf area $\left(32.98 \mathrm{dm}^{2}\right)$ and higher leaf specific weight $(0.4)$.
\end{abstract}

Index terms: Germination, shading, Fabaceae, Anacardiaceae.

(Recebido para publicação em 7 de novembro de 2003 e aprovado em 10 de agosto de 2005)

A cobertura florestal do Brasil tem sido intensamente agredida, especialmente nas regiões Sul, Sudeste, Centro-Oeste e Nordeste, onde remanescentes florestais nativos foram reduzidos a $8,6 \% ; 9,3 \% ; 15,3 \% \mathrm{e}$ $36,7 \%$, respectivamente, da área originalmente ocupada (FOWLER, 2000). No Pantanal, as formações arbóreas são exploradas para extração seletiva de madeira para a construção de currais, cercas e casas. Atualmente, as madeiras consideradas "de lei" estão se tornando escassas, havendo necessidade de se conhecer a estrutura e a composição florística das florestas, para embasar estudos futuros de manejo sustentável (SILVA et al., 1997).
Estudos de recuperação de áreas degradadas utilizam diferentes métodos silviculturais e diferentes espécies florestais, as quais são selecionadas segundo suas características ambientais em que vivem. A falta de conhecimento sobre as características fisiológicas das espécies utilizadas e a sua interação com as diferentes condições ecológicas, muitas vezes, levando alguns programas ao insucesso (PEREIRA et al., 2000). A habilidade de algumas espécies desenvolverem adequadamente em ambientes com diferentes disponibilidades luminosas, baseia-se na rapidez e eficiência de ajustar seus padrões de distribuição e seu

\footnotetext{
${ }^{1}$ Universidade Federal de Mato Grosso do Sul - NCA - Rodovia Dourados Ithaum, Km 12 - 79.804-970 - Dourados, MS- sscalon@ceud.ufms.br

${ }^{2}$ Centro Universitário da Grande Dourados/UNIGRAN - Dourados, MS - mara@unigran.br

${ }^{3}$ Universidade Estadual de Mato Grosso do Sul/UAA - Campus Aquidauana - Aquidauana - sscalon@ceud.ufms.br
} 
comportamento fisiológico, para maximizar a aquisição de recursos nesses ambientes (DIAS FILHO, 1997).

Informações precisas sobre procedimentos para produção de mudas de espécies arbóreas nativas no Brasil são muito escassas, existindo apenas para aquelas que detêm maior interesse econômico. Os viveiros tradicionais estão mais voltados à produção de um número reduzido de espécies, mais especificamente de Pinus e de Eucalipto (CARVALHO, 2000). Entretanto, observa-se na literatura uma crescente preocupação com a propagação de espécies nativas no País. Embora existam algumas informações sobre a aroeira do campo e o sombreiro, são escassos os dados sobre sua reprodução e o desenvolvimento das mudas.

A aroeira do campo (Schinus terebinthifolius Raddi - Anacardiaceae) é uma árvore ornamental, medindo de 5-10 m de altura, suas flores são melíferas e sua madeira é moderadamente pesada, resistente e de grande durabilidade natural, sendo utilizada para moirões, esteios, lenha e carvão. Suas sementes, quando semeadas logo após a colheita em canteiros a pleno sol e com substrato argiloso, apresentam emergência em 10-15 dias com uma taxa de germinação superior a 50\%. O desenvolvimento das plantas no campo é bastante rápido (LORENZI, 1998). Jain et al. (1995) observaram, em sua revisão, que essa espécie pode ser utilizada para combater processos inflamatórios como artrite reumatóide, asma e psoríase.

A sombreiro (Clitoria fairchildiana HowardFabaceae - Papilionoideae) é uma árvore ornamental, medindo de 6-12 m de altura. Sua madeira é moderadamente pesada e de baixa durabilidade sob condições naturais, sendo empregada em construção civil como divisórias internas, forros e para confecção de brinquedos e caixotaria. Segundo Lorenzi (1998), os frutos (vagens deiscentes) devem ser colhidos diretamente na árvore quando iniciarem a abertura espontânea, e secos ao sol para completar a abertura e liberação das sementes. A semeadura deve ser feita logo em seguida em canteiros semi-sombreados contendo substrato organo-argiloso. A emergência ocorre em 10-20 dias e a taxa de germinação é elevada . O desenvolvimento da muda é rápido, o mesmo ocorrendo com as plantas no campo que podem facilmente ultrapassar 2,5 $\mathrm{m}$ aos 2 anos de idade.

Como são poucos os dados na literatura sobre técnicas de cultivo dessas espécies, objetivou-se com este trabalho avaliar o crescimento inicial das mudas de aroeira do campo e sombreiro sob condições de sombreamento e de pleno sol.

Os frutos de aroeira do campo foram levemente esmagados e semeados sem despolpamento, diretamente em embalagens de polietileno $10 \times 20 \mathrm{~cm}$ contendo como substrato terra e adubo (NPK 4-14-8 - $2 \mathrm{Kg} / \mathrm{m}^{3}$ de solo). As sementes de sombreiro foram colhidas diretamente do chão e semeadas no mesmo substrato. Aos 60 dias após a emergência, as mudas foram transferidas e acondicionadas em canteiros sob sombrites de $70 \%$ e $50 \%$ de luz e sob luz plena.

O experimento foi conduzido em delineamento de blocos casualizados com quatro repetições de 20 mudas. Ao final do experimento, quando as mudas estavam com 123 dias de idade, foram realizadas as avaliações de altura $(\mathrm{cm})$, diâmetro $(\mathrm{cm})$, peso da matéria seca total $(\mathrm{PST}) \mathrm{em}$ gramas, área foliar (AF) em dm² medida com auxílio do medidor de área foliar LI Cor 3000, razão de peso foliar $(\mathrm{RPF}=$ Peso folha / Peso total em $\mathrm{g} / \mathrm{g}$ ); e peso específico de folha $\left(\mathrm{PEF}=\right.$ Peso seco da folha $/$ área folha em $\left.\mathrm{g} / \mathrm{dm}^{2}\right)$, segundo Benincasa (1988). Os resultados foram submetidos à análise de variância e as médias comparadas pelo teste de Tukey a $5 \%$ de probabilidade.

A altura média das mudas de aroeira do campo foi significativamente maior sob menor disponibilidade de luz $(50 \%)$ embora não tenha havido diferença significativa para o diâmetro de caule, o peso seco total apresentou menor valor nesse mesmo nível de sombreamento (TABELA 1).

Sob condições de pleno sol as mudas apresentaram menor altura, maior peso seco total. Poggiani et al. (1992) estudando os efeitos de sombreamento em plântulas de angico-branco (Piptadenia rigida Benth), guapuruvu (Schizolobium parahyba (Vell.) Blake) e coração de negro (Albizia lebbeck (L.) Benth), concluíram que essas espécies apresentaram um maior crescimento em altura nas condições de sombreamento do que a plena luz. Gajego et al. (2001) observaram que Maclura tinctoria (L.) D.Don ex. Steud não apresenta diferença significativa do diâmetro de caule sob diferentes condições de luz, entretanto as mudas apresentam maior altura sob sombreamentos de 30 e $50 \%$ e conseqüentemente menor razão raiz/parte aérea.

A razão de peso foliar representa a capacidade de translocação de fotoassimilados da parte aérea para o resto da planta e, quanto maior for esta razão, mais eficiente é a translocação, o que favorece o aumento no diâmetro. Em mudas crescendo sob sombreamento de 50\%, observase que a razão de peso foliar foi menor (TABELA 1).

$\mathrm{O}$ peso específico de folhas, que representa a espessura da folha (BENINCASA, 1988), foi significativamente menor e a área foliar maior, nas mudas sob pleno sol. Porém, analisando a área foliar, sugere-se que um auto sombreamento pode ter levado à proteção natural das próprias folhas. Farias et al. (1997) observaram maior área foliar sem aumento de 
matéria seca de mudas de cedrorana (Cedrelinga catenaeformis Ducke Ducke) sob 70\% de sombreamento, e sugerem que a baixa intensidade de luz provoca aumento da expansão foliar e redução da espessura das folhas.

As plântulas de angico branco apresentaram aumentos significativos do peso seco das folhas e da área foliar quando expostas a $80 \%$ de sombreamento. Um fator determinante na sobrevivência de uma espécie é sua adaptação a condições de alta ou baixa luminosidade. Em geral, os diferentes graus de luminosidade causam mudanças fisiológicas e morfológicas na planta, sendo o grau dessa adaptação ditado por características genéticas da planta em interação com o seu meio ambiente (MORAIS NETO et al., 2000).

Observa-se portanto, que a aroeira sob 50\% de luminosidade apresentou maior crescimento em altura, o que facilitou a absorção de luz, e pode representar uma certa intolerância a essa condição. Sob sombreamento moderado (70\% de luz), as mudas ainda apresentaram um bom desenvolvimento; entretanto, como um maior desenvolvimento da parte aérea, referente não somente à altura mas também ao diâmetro e área foliar, é desejado no processo de obtenção de mudas, parece que o cultivo inicial dessa espécie a pleno sol é o mais indicado.

Não houve diferença significativa na altura e na razão de peso foliar das mudas de sombreiro nas condições de luz estudada. (TABELA 2). Farias et al. (1997) observaram que a altura e o diâmetro do caule de cedrorana (Cedrelinga catenaeformis) também não foram influenciados pelos níveis de luz.
Sob condições de pleno sol, as mudas apresentaram altura e RPF semelhantes aos outros tratamentos, entretanto, o peso seco total e a área foliar foram significativamente menores a pleno sol e o diâmetro reduziu sob maior disponibilidade de luz.

O peso específico de folhas mantidas a pleno sol foi significativamente maior que nas mudas sob sombreamento, podendo estar relacionado com a menor área foliar apresentada pelas mudas a pleno sol.

As mudas de sombreiro não se desenvolveram satisfatoriamente nas condições de pleno sol, apresentando sob sombreamento, maior peso seco total, maior área foliar e tendência de maior diâmetro do colo. Assim, um regime de sombreamento moderado na fase inicial de crescimento, parece ser o mais indicado para essa espécie. Esses resultados corroboram aqueles observados por Portela et al. (2001) nos quais, as mudas de sombreiro apresentaram maior altura, incremento em comprimento e peso seco de raiz a pleno sol e maior crescimento de raiz a $30 \%$ de sombreamento. Os autores sugerem que maiores pesos de matéria seca tendem a indicar maior vigor das mudas. Assim, recomendam a produção das mudas dessa espécie sob sombreamento de $30 \%$ caso o plantio das mesmas seja imediato (6 meses) ou a pleno sol caso as mudas sejam estocadas.

Baseado nos resultados encontrados nesse trabalho, podemos concluir que as mudas de aroeira apresentam maior crescimento inicial a pleno sol enquanto as mudas de sombreiro sob condição de sombreamento.

TABELA 1 - Altura; diâmetro do colo; peso da matéria seca total (PST); área foliar (AF ); razão do peso foliar (RPF); peso específico de folha (PEF) de mudas de aroeira avaliada sob diferentes condições de luz. UNIGRAN, Dourados - MS. 2005.

\begin{tabular}{lcrrrr}
\hline \multicolumn{1}{c}{ Avaliações } & $\mathbf{5 0 \%}$ & $\begin{array}{c}\text { Disponibilidade de Luz } \\
\text { P0\% }\end{array}$ & Pleno Sol & CV \\
\hline Altura $(\mathrm{cm})$ & $51,42 \mathrm{a}$ & $41,75 \mathrm{~b}$ & 32,93 & $\mathrm{c}$ & 5,99 \\
Diâmetro $(\mathrm{mm})$ & $6,83 \mathrm{a}$ & $7,89 \mathrm{a}$ & $7,15 \mathrm{a}$ & 14,96 \\
PST $(\mathrm{g})$ & $61,69 \mathrm{~b}$ & $61,82 \mathrm{~b}$ & $74,66 \mathrm{a}$ & 1,09 \\
AF $\left(\mathrm{dm}^{2}\right)$ & $231,27 \mathrm{~b}$ & $203,47 \mathrm{c}$ & $1664,63 \mathrm{a}$ & 0,33 \\
RPF $(\mathrm{g} / \mathrm{g})$ & $0,53 \mathrm{a}$ & $0,55 \mathrm{a}$ & $0,56 \mathrm{a}$ & 1,52 \\
PEF $\left(\mathrm{g} / \mathrm{dm}^{2}\right)$ & $0,14 \mathrm{a}$ & $0,17 \mathrm{a}$ & $0,025 \mathrm{~b}$ & 1,21 \\
\hline
\end{tabular}

Médias seguidas de mesma letra minúscula na linha são estatisticamente iguais pelo teste de Tukey a 5\% de probabilidade.

Ciênc. agrotec., Lavras, v. 30, n. 1, p. 166-169, jan./fev., 2006 
TABELA 2 - Valores médios de altura; diâmetro; peso seco total (PST); área foliar (AF); razão do peso foliar (RPF); peso específico de folha (PEF) de mudas de sombreiro sob diferentes condições de luz. UNIGRAN, Dourados - MS. 2005.

\begin{tabular}{|c|c|c|c|c|}
\hline \multirow{2}{*}{ Avaliações } & \multicolumn{3}{|c|}{ Disponibilidade de Luz } & \multirow{2}{*}{$\begin{array}{c}\text { CV } \\
(\%)\end{array}$} \\
\hline & $50 \%$ & $70 \%$ & Pleno sol & \\
\hline Altura $(\mathrm{cm})$ & 15,92 a & $17,21 \mathrm{a}$ & $16,04 \mathrm{a}$ & 13,33 \\
\hline Diâmetro (mm) & 6,42 a & $5,63 \quad b$ & $5,32 \quad b$ & 7,27 \\
\hline $\operatorname{PST}(\mathrm{g})$ & $32,28 \quad b$ & 38,63 a & 26,22 & 1,20 \\
\hline $\mathrm{AF}\left(\mathrm{dm}^{2}\right)$ & $116,43 \quad b$ & 129,31 a & 32,98 & 0,33 \\
\hline $\mathrm{RPF}(\mathrm{g} / \mathrm{g})$ & $0,51 \mathrm{a}$ & $0,50 \mathrm{a}$ & $0,51 \mathrm{a}$ & 1,64 \\
\hline $\mathrm{PEF}\left(\mathrm{g} / \mathrm{dm}^{2}\right)$ & $0,14 \quad b$ & $0,15 \quad b$ & $0,4 \quad \mathrm{a}$ & 1,37 \\
\hline
\end{tabular}

Médias seguidas de mesma letra minúscula na linha são estatisticamente iguais pelo teste de Tukey a 5\% de probabilidade.

\section{REFERÊNCIAS BIBLIOGRÁFICAS}

BENINCASA, M. M. P. Análise de crescimento de plantas: noções básicas. Jaboticabal: FCAV-UNESP, 1988. 41 p.

CARVAlHO, P. E. R. Produção de mudas de espécies nativas por sementes e implantação de povoamentos. In:

Reflorestamento de propriedades rurais para fins produtivos e ambientais: um guia para ações municipais e regionais. Brasília, DF: EMBRAPA; Colombo, PR: EMBRAPA Florestas, 2000. 315 p.

DIAS FILHO, M. B. Physiological response of Solanum crinitum Lam. to contrasting light environments. Pesquisa Agropecuária Brasileira, Brasília, n. 32, p. 789-796, 1997.

FARIAS, V. C. C.; COSTA, S. S.; BATALHA, L. F. P. Análise de crescimento de mudas de cedrorana (Cedrelinga catenaeformis (Ducke) Ducke) cultivadas em condições de viveiro. Revista Brasileira de Sementes, Brasília, v. 19, n. 2, p. 193-200, 1997.

FOWLER, J. P. Superação de dormência e armazenamento de sementes de espécies florestais. In: GALVÃO, A. P. M. Reflorestamento de propriedades rurais para fins produtivos e ambientais: um guia para ações municipais e regionais. Colombo, PR: EMBRAPA/CNPF, 2000. p. 72-100.

GAJEGO, E. B.; ZANELA, S. M.; SOARES, A. M.; LIMA JÚNIOR, E. C.; ALVARENGA, A. A. Crescimento de plantas jovens de Maclura tinctoria (L.) D.Don ex. Steud e Hymeneae coubaril (L.) em diferentes condições de sombreamento. In: CONGRESSO NACIONAL DE FISIOLOGIA, 8., 2001, IlhéusBA. Resumos... Ilhéus: [s.n.], 2001. CD-ROM.

JAIN, M. K.; YU, J. M.; ROGERS, A. E.; SMITH, E. T. A.; BORGER, R. L.; OSTRANDER, A. L. Specific competitive inhibitor of secreted phospholipase A2 from berries of Schinus terebinthifolius. Phytochemistry, Oxford, v. 39, n. 3, p. 537-547, 1995.

LORENZI, H. Árvores brasileiras: manual de identificação e cultivo de plantas arbóreas nativas do Brasil. Nova Odessa: Plantarum, 1998. 352 p.

MORAIS NETO, S. P.; GONÇALVES, J. L. de M.; TAKAKI, M.; CENCI, S.; GONÇALVES, J. C. Crescimento de mudas de algumas espécies arbóreas que ocorrem na mata atlântica em função do nível de luminosidade. Revista Árvore, Viçosa, v. 24, n. 1, p. 35-45, 2000.

PEREIRA, J. A. A.; BOTELHO, S. A.; DAVIDE, A. C. Comportamento de três espécies florestais de rápido crescimento em diferentes sítios com vistas à recomposição de matas ciliares. In: CONGRESSO E EXPOSIÇÃ̃O INTERNACIONAL SOBRE FLORESTAS E MATAS CILIARES, 2000, Porto Seguro. Resumos técnicos... Porto Seguro: Forest, 2000. p. 72.

POGGIANI, F.; BRUNT, S.; BARBOSA, E. S. Q. Efeito do sombreamento sobre o crescimento das mudas de três espécies florestais. Revista do Instituto Florestal, São Paulo, v. 2, p. 564-569, 1992.

PORTELA, R. C. Q.; SILVA, I. L.; PIÑA-RODRIGUES, F. C. M. Crescimento inicial de mudas de Clitoria fairchildiana H. e Peltophorum dubium Spreng. Taub. em diferentes condições de sombreamento. Ciência Florestal, Santa Maria, v. 11, n. 2, p. 163-170, 2001.

SILVA, I. R.; FURTINI-NETO, A. E.; CURI, N.; VALE, F. R. Cescimento inicial de quatorze espécies florestais nativa em resposta à adubação potássica. Pesquisa Agropecuária Brasileira, Brasilia, v. 32, n. 2, p. 205-212, 1997. 